\title{
Investigating the Optical Properties of Mercury (Hg) Nanoparticles Using CryoSTEM-VEELS
}

\author{
D. H. Anjum ${ }^{*}$ and R. Sougrat ${ }^{* *}$ \\ *FEI Company, 5350 NE Dawson Creek Dr., Hillsboro, OR, 97124 \\ **4700 King Abdullah University of Science \& Technology (KAUST), Thuwal, 23955, Kingdom of \\ Saudi Arabia (KSA)
}

Optical properties of $\mathrm{Hg}$ metal had been a topic of discussion in the past due to some discrepancies generated by the predictions of free electron gas (Drude) model and their experimental observations $[1,2,3]$. Little new insights were brought in understanding these properties for both solid and liquid phases of $\mathrm{Hg}$. Hence a renewed effort was attempted in this report to investigate the optical properties of $\mathrm{Hg}$ using the technique of scanning transmission electron microscopy and valence electron energy loss (STEM-VEELS). STEM-VEELS spectrum imaging (SI) datasets were acquired from $\mathrm{Hg}$ nanoparticles of different sizes sitting on a carbon film at the temperature of $100 \mathrm{~K}$ and at the vacuum level below $0.1 \mathrm{mPa}$. Entire experiments were carried out using a Titan TEM (FEI Company, Hillsboro, OR) operating at $300 \mathrm{kV}$ and equipped with an energy filter (Tridiem GIF from Gatan, Inc.). Furthermore, SI datasets were acquired with $0.7 \mathrm{~nm}$ and $1.4 \mathrm{eV}$ spatial and energy resolutions, respectively. Kramers-Kronig (KK) analysis was then applied to single scattering data (SSD) after the plural scattering contribution was first removed. Various optical parameters such as dielectric function $\left(\varepsilon=\varepsilon_{1}+\varepsilon_{\mathrm{b}}+\mathrm{i} \varepsilon_{2}\right)$ of $\mathrm{Hg}$ nanoparticles in solid phase were determined and discussed as a function of energy loss.

High angle annular dark-field STEM (HAADF-STEM) micrographs of various size nanoparticles are shown in Fig.1. A STEM-EELS SI dataset was acquired along a line across each nanoparticle of Fig.1. Real $\left(\varepsilon_{1}\right)$ and imaginary $\left(\varepsilon_{2}\right)$ parts of $\varepsilon$ were determined from the central regions $(5 \mathrm{~nm})$ of each $\mathrm{Hg}$ nanoparticle using the KK analysis (Fig. 2A and Fig. 2B). The bulk Plasmon energy $\left(E_{p}=\hbar \omega_{p}\right)$ is usually determined as the energy loss value at which $\varepsilon_{1}$ turns zero. According to Drude model, $\mathrm{E}_{\mathrm{p}}$ was predicted to be $10.9 \mathrm{eV}$ for $\mathrm{Hg}$ metal [1]. However, its exact measurement had always been subject to discussion $[1,2]$. Interestingly our observations show that $\mathrm{E}_{\mathrm{p}}$ energy of $75 \mathrm{~nm}$ and $55 \mathrm{~nm}$ size nanoparticles was close to the one predicted by Drude model (Fig.2A). This implies that electronic behavior for these two particles is nearly like a free electron gas. On the other hand, a strong coupling of interband transitions $\left(\varepsilon_{\mathrm{b}}\right)$ was noticed for $30 \mathrm{~nm}$ and $23 \mathrm{~nm}$ size nanoparticles. In fact, the effect of these interband transitions was much stronger in the case of $20 \mathrm{~nm}$ size nanoparticle as its $\varepsilon_{1}$ was pushed above zero at $\sim 4.5 \mathrm{eV}$ energy loss possibly due to s-d type transitions (Fig. 2A). Whereas for $30 \mathrm{~nm}$ size nanoparticle Ep had been blue-shifted to $14 \mathrm{eV}$ energy value but contribution of s-d type transitions at $4.5 \mathrm{eV}$ was found to quite less unlike the $23 \mathrm{~nm}$ size particles. Interband transitions occurred quite significantly at the surfaces $(3 \mathrm{~nm})$ of all nanoparticles as shown in Fig.3A and Fig. 3B by the multiple oscillations of $\varepsilon_{1}$ and $\varepsilon_{2}$ in the range of $2-8 \mathrm{eV}$ energy loss. It can also be noted in Fig. 3 that only $75 \mathrm{~nm}$ size particle exhibited the surface plasmon energy of $\sim 6.3 \mathrm{eV}$ as predicted by Drude model [1] and the rest of smaller size nanoparticles showed strong surface effects. Finally the bulk moduli $\left(\mathrm{B}_{\mathrm{m}}\right)$ could be calculated for these nanoparticles since it is related $E_{p}$ energy [4]. The value of $B_{m}$ for $75 \mathrm{~nm}$ size nanoparticle turned out to be $41 \mathrm{GPa}$. This is higher than the already reported value of $25 \mathrm{GPa}$ at the temperature and pressure of $20^{\circ} \mathrm{C}$ and 19 $\mathrm{MPa}$, respectively [5]. An apparent difference of $\sim 15 \mathrm{GPa}$ between the two values could be due to 
having quite different temperature and pressure conditions between the two set experiments namely in the TEM column than in the ref. [5].

In conclusion, STEM-VEELS technique offered the opportunity to revisiting the optical properties of $\mathrm{Hg}$ metal at the nanoscale length. Nonetheless a lot more work is still remained to be understood both on experimental and theoretical aspects of EELS data from these $\mathrm{Hg}$ nanoparticles.
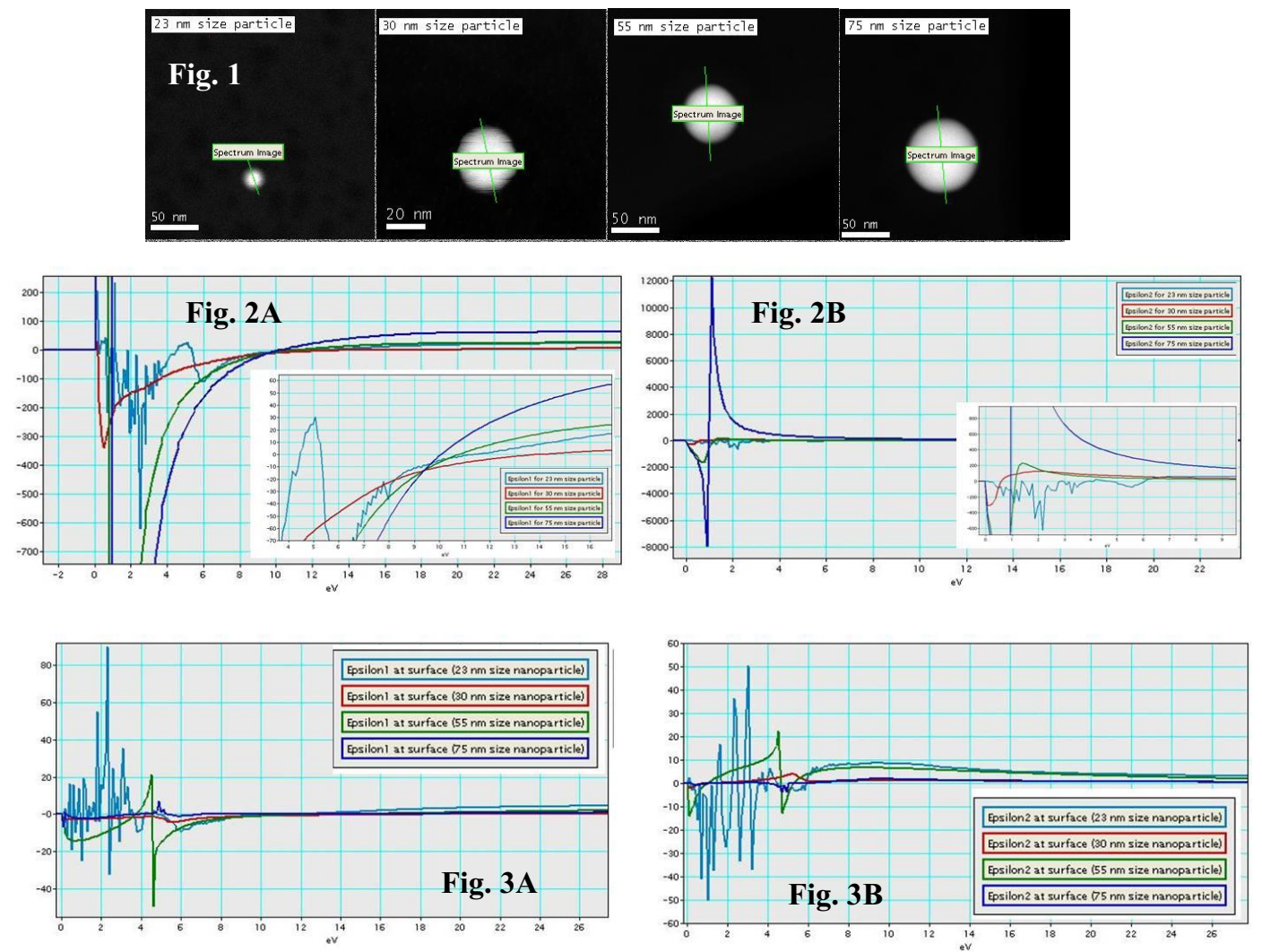

Fig.1: HAADF-STEM micrographs of $\mathrm{Hg}$ nanoparticles examined using STEM-VEELS. Each SI dataset was acquired with $\sim 450 \mathrm{e}^{-} / \AA^{2}$ dose along scan-lines (green) shown on each micrograph. The size of each nanoparticle is indicated.

Fig. 2 A-B: Real $\left(\varepsilon_{1}\right)$ and imaginary $\left(\varepsilon_{2}\right)$ parts of dielectric function of $\mathrm{Hg}$ nanoparticles integrated from their central regions $(5 \mathrm{~nm})$ plotted as function of energy loss. The insets are zoomed areas for highlighting their behavior as a function of energy loss.

Fig.3 A-B: Real $\left(\varepsilon_{1}\right)$ and imaginary $\left(\varepsilon_{2}\right)$ parts of dielectric function of $\mathrm{Hg}$ nanoparticles integrated from their surfaces $(3 \mathrm{~nm})$ plotted as function of energy loss.

\section{References}

[1] A. R. Krauss et.al, Phy. Rev. B, 13 (8), 3419-3423 (1976).

[2] T. Inagaki et al., Phy. Rev. B, 23 (10), 5246-5262 (1981).

[3] W. E. Mueller et al., Phy. Rev. Lett., 23 (18), 1037-1038 (1969).

[4] J. M. Howe et al., J. Micro. 53 (4), 339-351 (2004).

[5] A. T. J. Hayward, J. Phys. D, vol. 4, 951-955 (1971).

[6] Authors thank Dr. Ioannis Alexandrou (FEI Company) for useful discussion on KK analysis. 